\title{
Experimental Analysis of Polarization Dependence of Ultrafast Gain Dynamics in SOA's
}

\author{
B.F. Kennedy ${ }^{a}$, P. Landais ${ }^{a}$ and A.L. Bradley ${ }^{b}$ \\ ${ }^{a}$ Research Institute for Networks and Communications Engineering, School of Electronic \\ Engineering, Dublin City University, Dublin 9, Ireland \\ ${ }^{b}$ Semiconductor Optronics Group, Physics Department, Trinity College, Dublin 2, Ireland
}

\begin{abstract}
Nonlinear polarization rotation in semiconductor optical amplifiers has been the focus of a lot of work in the past decade. A lot of research has been devoted to this phenomenon due to its possible use in all-optical switching. It has been mentioned as a possible competitor to such established switching techniques as cross-gain modulation, cross-phase modulation and four-wave mixing. The speed at which the switching can be performed is determined by the gain dynamics in the device. So far the majority of the work has focused on switching due to the relatively slow carrier density recombination, which limits the switching to the order of tens of gigahertz. If the polarization dependence of ultrafast gain mechanisms such as carrier heating and spectral hole burning can be identified and measured then there is the possibility to increase the switching speed obtainable using this process into the terahertz range. In order to further the understanding of the polarization dependence of the gain of a bulk SOA under tensile strain and to determine the plausibility of ultrafast all-optical switching using nonlinear polarization rotation an experiment is presented based on a four-wave mixing technique.
\end{abstract}

Keywords: nonlinear polarization rotation, semiconductor optical amplifier, all-optical switching, ultrafast gain mechanisms, four-wave mixing.

\section{INTRODUCTION TO NONLINEAR POLARIZATION ROTATION}

The semiconductor optical amplifier (SOA) is receiving considerable attention as a fundamental component in all-optical signal processing, for example as a switching and wavelength conversion component due to its large bandwidth, high saturation power and low production cost. ${ }^{1}$ Several techniques have been developed in order to implement the SOA in such processes. The main techniques to date have been cross-gain modulation, crossphase modulation and four-wave mixing (FWM). ${ }^{1}$ Another technique that has begun to receive a lot of attention as an alternative technique to perform all-optical switching is the nonlinear polarization rotation (NPR) in an $\mathrm{SOA}^{2}$-. ${ }^{4}$ This technique is based on the fact that a signal injected into an SOA with a linear polarization appears at the output of the device with a rotated polarization. The degree of the rotation in the state of polarization can be controlled by the intensity of the injected signal, ${ }^{5}{ }^{6}$ There are several physical effects that lead to NPR in the SOA. The first studied was the inherent birefringence in the device due to the waveguide typically used in the SOA having an asymmetric geometry. This leads to light travelling at different speeds for signals polarized along the TE and TM axes' of the device. There is also a polarization dependence of the confinement factors in the device also due to issues of waveguiding. These effects generally lead to the TE gain being greater than the TM gain. One method commonly used to equalize the gains is to introduce a tensile strain to the active region of the device in order to enhance the TM gain over the TE gain and in this way to achieve a polarization independence in the device. This is effective in reducing the polarization dependence, although complete polarization independence has never been achieved. This polarization dependence of the gain is another effect that is crucial to the understanding of the NPR in SOA's. Much of the work on NPR has so far concentrated on the polarization dependence due to the slow carrier recovery time, ${ }^{7}$ but for the tensile-strained case it is expected that there will also be a polarization dependence of such ultra-fast processes

Further author information: (Send correspondence to B.F. Kennedy)

E-mail: kennedyb@eeng.dcu.ie, Telephone: +35317005884

Opto-Ireland 2005: Optoelectronics, Photonic Devices, and Optical Networks, edited by 185 
as carrier heating $(\mathrm{CH})$, spectral hole burning (SHB) and two-photon absorption (TPA). ${ }^{8}$ The reason for the polarization dependence of these processes is due to the asymmetry introduced into the band structure by the tensile strain. This is because the light amplified along the TE and TM axes of the SOA originate from different resevoirs of holes in the valence band with different populations in the case of a strained waveguide. ${ }^{4}$ It has been shown that for pulses below approximately 10ps the ultrafast gain dynamics are of primary importance, ${ }^{9}$ therefore for pulses of this order we can expect additional contributions to the NPR effect due to the ultra-fast gain dynamics.

\section{SOA UNDER TEST}

The SOA under test is a commercially available component. It is manufactured by Alcatel under the reference A1901. It is a tensile strained bulk amplifier structure with a $300 \mu \mathrm{m}$ central active waveguide, terminated by two $150 \mu \mathrm{m}$ lateral active tapered regions and $50 \mu \mathrm{m}$ lateral passive regions. For a bias of $200 \mathrm{~mA}$, the amplified spontaneous emission (ASE) collected by a $0.3 \mathrm{NA}$ GRIN lensed fibre is $2 \mathrm{~mW}$. The lensed fibre is required as the device was not a pigtailed device, making control of the polarization of the injected signal possible. The ASE spectrum can be seen in Fig. 1 for a bias current of $200 \mathrm{~mA}$. The peak of the ASE occurs at $1515 \mathrm{~nm}$. The $3 \mathrm{~dB}$ width of the spectrum is over $70 \mathrm{~nm}$. The wide ASE spectrum is related to the relatively short length of the device. The LI curve of the SOA is shown in Fig 2. Below $50 \mathrm{~mA}$ the SOA acts as a light emitting diode, above this current the spontanoeus emission is amplified and guided in the active region.

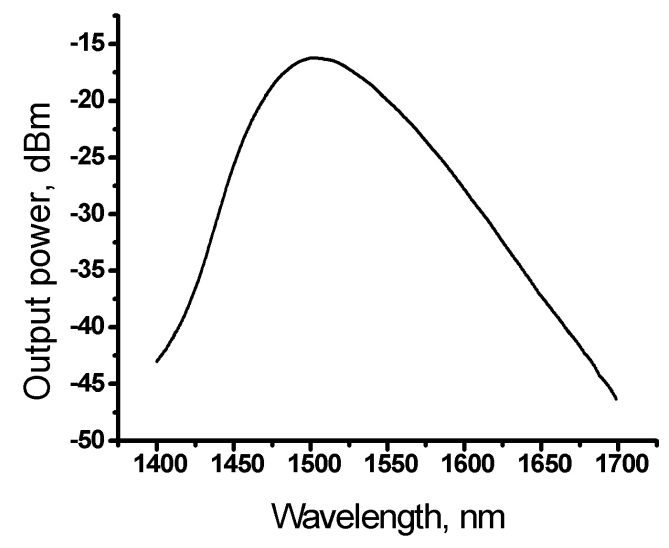

Figure 1. ASE spectrum of the device used in the experiment

\section{GAIN DYNAMICS IN THE SOA}

When an optical beam is injected into an amplifier, electrons in excited states are depleted due to the stimulated emission that leads to the generation of photons. This depletion of the carriers leads to a reduction of the gain. There is also a modulation of the refractive index, which is determined by the linewidth enhancement factor (LEF), also known as the Henry factor, $\alpha$. After depletion of the carriers there is a recovery of the gain to the initial undepleted value. This recovery is determined by several processes with a certain timeframe associated with each process. The main processes involved in the recovery of the gain to its initial value are $\mathrm{CDP}, \mathrm{CH}, \mathrm{SHB}$ and TPA. Much of the research on all-optical signal processing has focussed on exploiting the CDP to perform the processing, but this limits the speed of operation to the order of tens of gigahertz, due to the relatively long timeframe associated with this process. If the faster processes of $\mathrm{CH}$, SHB and TPA can be used instead of CDP, much faster processing speeds could be achieved. The experiment presented has a resolution on the sub-hundred femtosecond scale that is sufficient to account for CDP, $\mathrm{CH}$ and SHB but is not powerful enough to account for such instantaneous effects such as TPA and and optical Kerr effects. A diagram of the evolution of the carrier distribution as a function of energy is given in Fig. 3, showing the different processes that occur at different timeframes. 


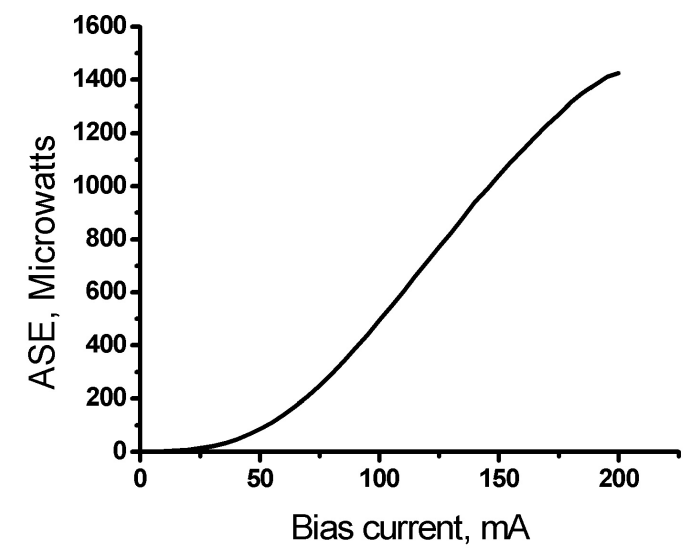

Figure 2. LI curve of the SOA

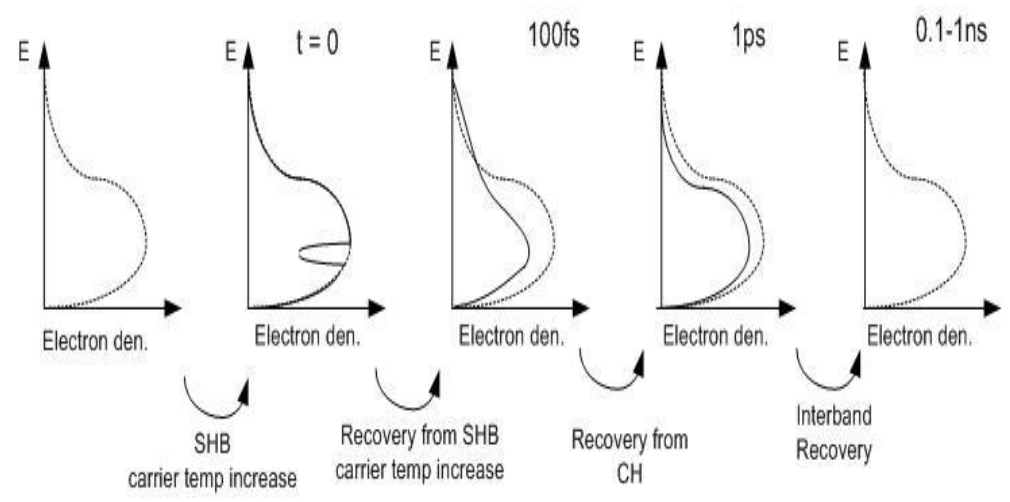

Figure 3. Evolution of carrier distribution in the active region of a bulk SOA. Following stimulated emission due to a monochromatic optical signal the distribution recovers to equlibrium on several different timeframes due to the different gain processes.

\subsection{CARRIER DENSITY PULSATIONS}

The timeframe associated with this process is related to the relaxation time required for the transition of carriers from the valence band to the conduction band. It is the well-known interband relaxation time, that is described by the equation ${ }^{10}$ :

$$
\tau_{s}^{-1}=\partial R(N)=A+2 B N+3 C N^{2}
$$

where $\tau_{s}$ is the inter-band carrier lifetime, $R(N)$ is the total carrier recombination rate, $A$ is the SchockleyRead-Hall leakage coefficient, $B$ is the bimolecular radiative rate coeffiecient and $C$ is the term related to the Auger recombination rate. $N$ describes the carrier density. $\tau_{s}$ decreases for increasing SOA length, bias current and injected optical power. Previous experiments have employed a holding beam to reduce the recombination time. ${ }^{11}$ Typical times associated with this process are in the order of hundreds of picoseconds, the exact value varies between devices. With the use of a holding beam recovery times as low as 10ps have been achieved. The $\mathrm{LEF}$ for the interband recombination is given by the following expression:

$$
\alpha_{c d p}=\frac{-4 \pi}{\lambda} \frac{\frac{\partial n}{\partial N}}{\frac{\partial g_{m}}{\partial N}}
$$


where $n$ is the refractive index, $N$ is the carrier density and $g_{m}$ is the material gain. This expression is a measure of the ratio between the rate of change of refractive index with respect to carrier density and the rate of change of material gain with respect to carrier density. It is a magnitude describing the modulation of the refractive index due to the inter band transitions.

\subsection{CARRIER HEATING}

When an optical signal is injected into an SOA, carriers at the energy corresponding to the signal are depleted, leading to SHB. The carriers that are removed by this process are generally the carriers with the least energy, they are referred to as 'cold carriers'. The removal of these carriers leads to the increase of the average energy of the remaining carriers after scattering has removed the spectral hole. This leads to carriers heating. Carrier heating due to stimulated emission is not the only cause of this effect. Free-carrier absortion (FCA) is also a contributing factor. FCA, also known as the plasma effect, is a process where an electron absorbs a photon within the conduction or valence band and transits to a higher energy level. These heated carrier distributions cool to the lattice temperature due to the emission of optical phonons, which are mechanical vibrations. The timeframe associated with this cooling is approxiamtely 1 ps although this value is dependent on the particular device under test. The recovery from $\mathrm{CH}$ is due to carrier-phonon scattering. The LEF originating from $\mathrm{CH}$ is given by:

$$
\alpha_{c h}=\frac{-4 \pi}{\lambda} \frac{\frac{\partial n}{\partial T_{c}}}{\frac{\partial g_{m}}{\partial T_{c}}}
$$

where $n$ is the refractive index, $T_{c}$ is the carrier temperature in the conduction band and $g$ is the modal gain.

\subsection{SPECTRAL HOLE BURNING}

SHB refers to a localized reduction in the number of carriers at the transition energies. The depth of the spectral hole is dependent on the amount of transitions of carriers from the conduction to the valence band. These transitions will be different for TE and TM polarization in a strained waveguide due to the loss of degeneracy in the valence band. The LEF for SHB is considered to be 0 because a spectral hole symmetrically depletes the gain around the emission wavelength, in such a situation no refractive index change results. ${ }^{12}$ Therefore there is no self-phase modulation (SPM) originating from the SHB. The timeframe associate with SHB is generally around $100 \mathrm{fs}$, although this value is variable for different devices. The recovery from SHB is due to carrier-carrier scattering.

\subsection{OTHER ULTRAFAST PROCESSES}

Other ultrafast processes such as TPA and optical Kerr effects are considered instantaneous, occuring at timeframes below SHB. These effects are not considered in this experiment.

\section{POLARIZATION DEPENDENCE OF GAIN DYNAMICS}

The reason for a polarization dependence of the gain dynamics in the SOA originates in the band structure. For the case of an unstrained waveguide there is complete symmetry in the gain of the device and there is no polarization dependence of the material gain. This situation is represented in Fig. 4(a). The valence band (VB) may be sub-divided into a heavy-hole ( $\mathrm{HH}$ ) band and a light-hole ( $\mathrm{LH}$ ) band, where the TE transitions predominantly occur with the heavy holes and the TM transitions occur predominantly with the light-holes. Because of the fact that the overall device gain is generally larger for the case of TE polarization due to issues of waveguiding, a technique generally used is to introduce tensile strain to enhance the LH transitions, and therefore the TM transitions, in order to minimize the variation in gain between TE and TM axes. ${ }^{13}$ This is effective and although complete polarization insensitivity has never been achieved, it is an effective technique in compensating for the higher TE gain. The effect of introducing a tensile strain to the device is shown in Fig. 4(b). In terms of the gain dynamics in the device, the situation under tensile strain is that there are effectively two material gains in the device which couple to two different resevoirs of holes. This is due to the degeneracy being removed from the valence band. The result is that there are also two different gain dynamics in the device. 
A polarization dependency may be expected due to the mixing of the two gain dynamics. If a signal is injected off-axis, i.e. with a combination of TE and TM light then the amount of light which couples to each mode will determine the overall time constant associated with each process.

a)

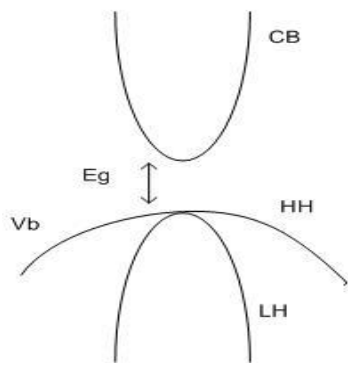

b)

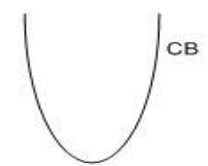

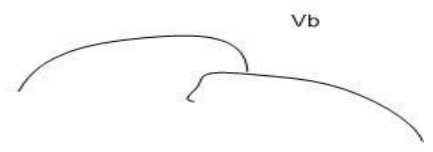

Figure 4. Band structure for (a) unstrained and (b) tensile strained semiconductor material

The polarization dependence of the interband gain due to the CDP was found experimentally. The HakkiPaoli technique ${ }^{14}$ was used to find this gain. The gain is determined from the ASE modulation depth. A polarizer was placed after the SOA in order to collect the ASE along orthogonal axes of the device. The gain found along TE and TM axes as a function of wavelength is shown in Fig. 5. It should be stressed that the gain calculated using this technique is the modal or device gain not the material gain. From Fig. 5 it can be seen that there is a discrepancy of approximately $2 \mathrm{~dB}$ between the TE and TM gain over the wavelength range considered.

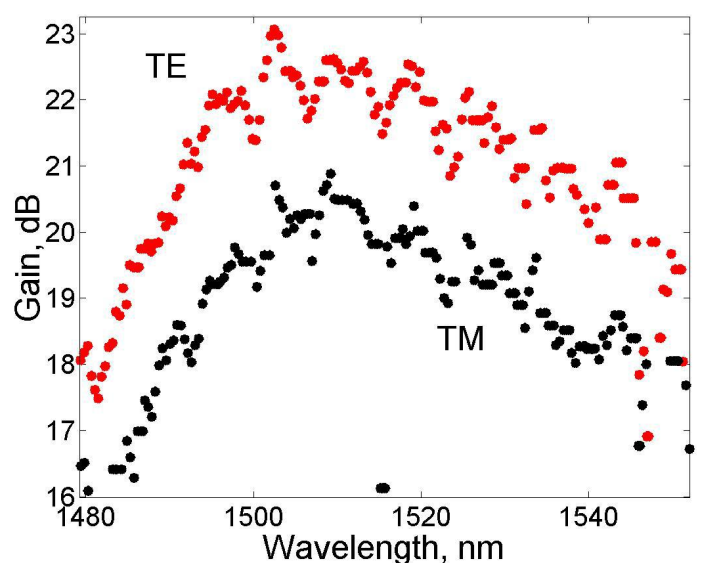

Figure 5. TE and TM gains found experimentally using the Hakki-Paoli technique

\section{PRINCIPLE OF FOUR-WAVE MIXING}

This experiment is based on FWM in the SOA. ${ }^{15}$ The principle of FWM is that a strong pump at an angular frequency $\omega_{0}$ interacts with a second weaker probe signal at an angular frequency $\omega_{1}$ to produce a conjugate signal at an angular frequency given by $\omega_{2}=2 \omega_{0}-\omega_{1}$, i.e. the new frequency components are generated on either side of the injected signals with a spacing determined by the beat frequency. The pump and probe signals must be co-polarized in order to generate the beating required for four-wave mixing. This beating of the pump and probe signals leads to a modulation of various parameters associated with the dynamic gain in the device. The particular gain processes that are modulated depends on the frequency of the generated conjugate wave, which in turn depends on the frequency detuning between the pump and the probe. The four-wave mixing parameter that is recorded to tell which gain parameter is being modulated is the conversion efficiency, defined as the conjugate power divided by the probe power, $P_{\text {conj }} / P_{\text {probe }}$ at the output of the SOA. For small detunings, 
corresponding to relatively large timescales, CDP is the strongest effect on the conversion efficiency. As the detuning is increased the significance of the CDP is reduced due to the relatively large timeframe associated with this process, and at certain frequencies other effects start to become important, namely $\mathrm{CH}$ and SHB, which have been reported to occur on timescales of approximately 1 ps and 100fs respectively. ${ }^{16}$ Other effects such as two photon absorption (TPA) and ultrafast kerr effects happen on much faster timescales of less than $100 \mathrm{fs} .{ }^{16}$ This degree of resolution is beyond the scope of this work. The generated conjugate signal can appear at a lower frequency than the pump and probe, down conversion, or at higher frequencies than the pump and probe, up conversion. For the conjugate signals generated in this experiment all the conversions were down conversions due to the fact that the impact of each of the processes on the efficiency was much more pronounced for down conversion. As well as providing insight into the different gain processes occuring in SOA's the FWM technique can be used to perform all-optical signal processing in its own right. For wavelength conversion, for example, a typical experimental setup is to have the data to be converted on the probe signal and the pump represented by a strong CW signal. If the data signal is small enough not to modulate the gain of the device then the data will be converted to a new wavelength, determined by the frequency spacing between the pump and the probe.

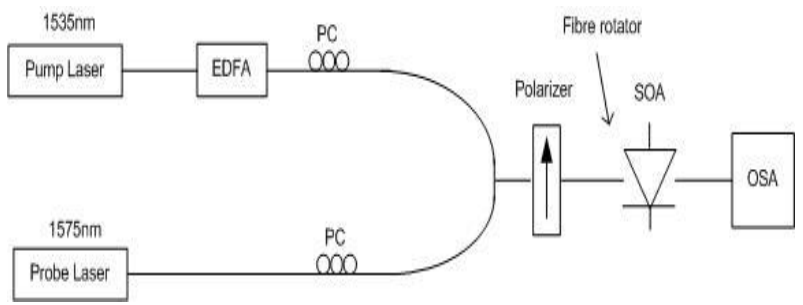

Figure 6. Experimental setup for FWM experiment

\section{EXPERIMENTAL SETUP AND RESULTS}

The experimental setup is shown in Fig. 6. Two external cavity tunable lasers are used to perform the four-wave mixing. The pump wavelength of $1535 \mathrm{~nm}$ is within the $3 \mathrm{~dB}$ gain wavelength of the device. Ideally it would be preferable to have the pump situated closer to the gain peak of the device, which occurs at $1515 \mathrm{~nm}$, but there is a restriction due to the Erbium Doped Fibre Amplifier (EDFA) which does not amplify significantly below $1535 \mathrm{~nm}$. The wavelength of the probe is varied from $1535 \mathrm{~nm}$ to $1575 \mathrm{~nm}$ in steps as low as $0.1 \mathrm{~nm}$. The polarization of both the pump and the probe are controlled using a polarization controller (PC) and a polarizer. The polarizer ensures that both signals are co-polarized, a requirement for FWM, as well as ensuring that the light is injected along the axis of the polarization maintaining (PM) lensed fibre that is used to couple the light into the SOA. The PC's are used to control the amount of light polarized along the axis of the PM fibre. The intensities of both the pump and probe are kept constant as a function of polarization. An optical spectrum analyser (OSA) with a resolution bandwidth of $0.06 \mathrm{~nm}$ is used to observe the spectra at the output of the device. An EDFA is used to amplify the pump signal as it must have a strong intensity in order to modulate the gain of the device. The pump power is kept constant at $9 \mathrm{dBm}$ for all experiments whilst the probe power is constant at $-4 \mathrm{dBm}$. This difference of $13 \mathrm{~dB}$ is close to the optimum difference used by other groups to produce the best results. ${ }^{16}$ The polarization of the co-polarized pump and probe is varied with respect to the axis of the device by physically rotating the fibre. This is easily achieved by means of a rotating mount which holds the fibre. The experimental efficiency data is used to extract the parameters of interest by fitting the curve with an equation for the efficiency, $\rho .{ }^{17}$

$$
\rho=S_{0}^{2}(L)\left|f_{c d p}(\Omega)+f_{c h}(\Omega)+f_{s h b}(\Omega)\right|^{2}
$$

where the contributions from carrier density pulsation $\left(f_{c d p}\right)$, carrier heating $\left(f_{c h}\right)$ and spectral-hole burning $\left(f_{s h b}\right)$ are defined as follows ${ }^{17}$ :

$$
\begin{gathered}
f_{c d p}(\Omega)=-\frac{1}{S_{s a t}} \frac{1-j \alpha_{c d p}}{2\left(1-j \Omega \tau_{c d p}\right)} \\
f_{c h}(\Omega)=-\frac{1}{S_{c h}} \frac{1-j \alpha_{c h}}{\left(1-j \alpha \tau_{c h}\right)\left(1-j \alpha \tau_{s h b}\right)}
\end{gathered}
$$




$$
f_{s h b}(\Omega)=-\frac{1}{S_{s h b}} \frac{1-j \alpha_{s h b}}{1-j \Omega \tau_{s h b}}
$$

the $\alpha_{i}$ parameters refer to the linewidth enhancement factors, the $\tau_{i}$ parameters refer to the recovery times and the $S_{i}$ parameters refer to the characteristic power associated with each process.

The angular frequency detuning is represented by $\Omega$. A program was developed to minimise the error between the experimental data and this mathematical expression where the aforementioned parameters were set as variables. This fitting was performed for the following angles of polarization: $0^{\circ}, 30^{\circ}, 45^{\circ}, 60^{\circ}$ and $90^{\circ}$. The accuracy of this fitting program may be gauged by considering the variance between the experimental data and the fitted data which is $0.0049,0.0079,0.0024,0.0037$ and 0.0055 , respectively, at the angles of polarization considered. The experimental data and fitted curve for the efficiency as a function of detuning are plotted in Fig. 7 for an angle of $60^{\circ}$, together with the calculated contribution from each of the gain processes considered. It can be seen that the CDP decreases by approximately $20 \mathrm{~dB} /$ decade, and that the ultrafast effects become more important as the detuning is increased.

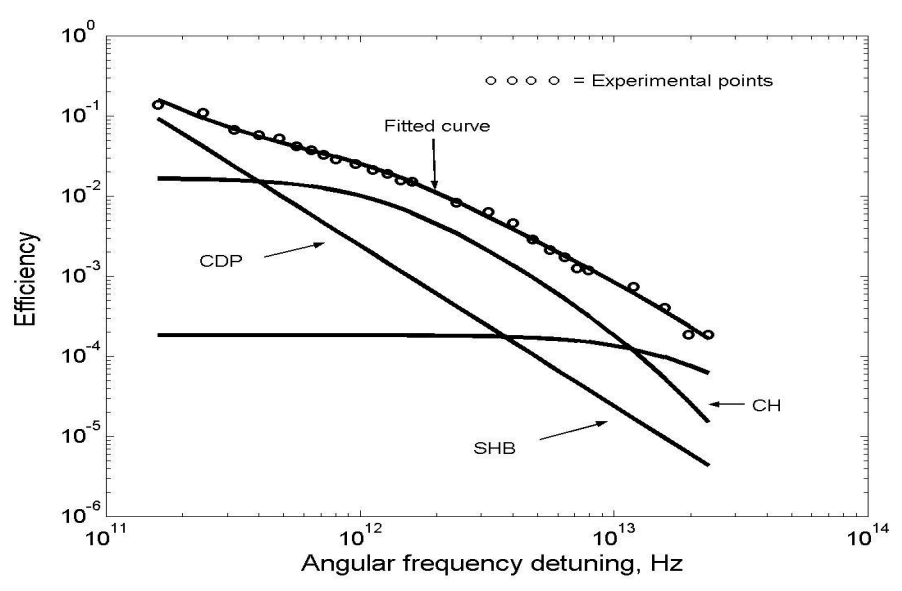

Figure 7. Experimental data used and curves extracted from fitting for different gain processes

\section{DISCUSSION}

The extracted data for each angle of polarizations considered are represented in Table 1. Before analysing these data it should be noted that $0^{\circ}$ corressponds to the TE axis and $90^{\circ}$ corressponds to the TM axis of the device. It can be seen from this table that there are large variations in the timeframes at which each of these processes occurs as a function of polarization. The time associated with the CDP varies from a minimum of $68.1 \mathrm{ps}$ at $0^{\circ}$ to $135 \mathrm{ps}$ at $90^{\circ}$. The longer carrier recovery time for the TM axis is due to the fact that for a bulk tensile strained device there are more TM transitions than TE transitions due to the shifting of the subbands in the valence band. The light holes, which give rise to the TM transitions are favoured under such strain. The result of this is a larger TM gain and so a greater depletion of carriers for light polarized along the TM axis. ${ }^{4}$ So TM light results in a lower carrier density population, this leads to a higher carrier recovery time due to the fact that carrier lifetime is inversely proportional to carrier density. ${ }^{10}$ Recovery times between $135 \mathrm{ps}$ at the maximum (TM) and 68.1ps at the minimum (TE) are recorded at $30^{\circ}, 45^{\circ}$ and $60^{\circ}$, this is due to the fact that the recovery at these angles have both a TE and a TM component. $\alpha_{c d p}$, the Henry factor due to inter-band recombinations, also varies from a value of 3.2 at $0^{\circ}(\mathrm{TE})$ to 6.34 at $60^{\circ}(\mathrm{TM})$. This result is not consistent with the work presented in ${ }^{18}$ which states that the Henry factor increases for increasing carrier density. As already explained there is a higher carrier density for TE polarization. With reference to $\alpha_{c d p}$, as the rate of change of gain with respect to carriers, $\frac{\partial g_{m}}{\partial N}$, will increase as the angle tends towards TM, the results from Table 1 indicate that the rate of change of refractive index with respect to the carriers $\frac{\partial n}{\partial N}$ will increase at a higher rate. This results in the increase of $\alpha_{c d p}$ as the angle is varied from TE to TM. The result when the angle of polarization is 
Table 1. Extracted Parameters

\begin{tabular}{|l|l|l|l|l|l|}
\hline & 0 deg & 30 deg & $45 \mathrm{deg}$ & $60 \mathrm{deg}$ & $90 \mathrm{deg}$ \\
\hline$\alpha, \mathrm{CDP}$ & 3.268 & 5.08 & 5.82 & 6.34 & 5.34 \\
$\tau, \mathrm{CDP}(\mathrm{ps})$ & 61.8 & 71.8 & 91.4 & 98.6 & 135 \\
$\alpha, \mathrm{CH}$ & 1.11 & 1.53 & 1.61 & 1.95 & 3.48 \\
$\tau, \mathrm{CH}(\mathrm{fs})$ & 534 & 717 & 713 & 819 & 668 \\
$\tau, \mathrm{SHB}(\mathrm{fs})$ & 31.3 & 65.4 & 39 & 59.8 & 52.7 \\
\hline
\end{tabular}

along the TM axis of the device goes against this explanation and further experiments are needed to justify this result. In terms of $\mathrm{CH}$ it can be observed that the longest time associated with this process occurs at $60^{\circ}$. This is a somewhat suprising result in that it would be expected that the longest time for this process would be at $90^{\circ}$ $(\mathrm{TM})$, as this is the polarization leading to the most transitions for this sample. The fact that there are several contributing factors to $\mathrm{CH}$ make the analysis of these results complex. However, some consistency with this result has been found with the same sample. ${ }^{19}$ In this experiment the largest impact of $\mathrm{CH}$ on $2 \mathrm{ps}$ pulses was found at $60^{\circ}$, indicating that the longest recovery time associated with this process was at $60^{\circ}$. A polarization dependence of the Henry factor associated with carrier heating, $\alpha_{c h}$, has been reported in the literature. ${ }^{20} \alpha_{c h}$ is greatest for TM polarization, which is the polarization for which $\mathrm{CH}$ due to removal of cold carriers will be greatest, due to the dominance of TM transitions. The contribution due to free-carrier absorption (FCA) is harder to determine and an experiment similiar to Murata et al. ${ }^{20}$ would need to be performed to determine the polarization dependence of this effect. From Table 1 it can be inferred that the polarization dependence of the FCA is either negligible or follows a similiar pattern to the removal of cold carriers.

The removal of cold carriers leads to a reduction of the $\frac{\partial g}{\partial T_{c}}$ term in the expression for $\alpha_{c h}$, due to the increased change in the temperature, resulting in an increase in $\alpha_{c h}$ as the angle of polarization is varied from TE to TM. It has been reported ${ }^{21}$ that $\mathrm{CH}$ has a negligible effect on the phase variation of a signal injected into the SOA. This is due to $\alpha_{c h}$ generally being much smaller, 5-10 times smaller, than $\alpha_{c d p}$. From Table 1 it can be seen that this is the case for an angle of $0^{\circ}$, but as the angle is increased the magnitude of $\alpha_{c h}$ increases and there will be a significant contribution to the phase due to this parameter. The polarization dependence of the recovery from SHB is not easily explained. It would be expected that the longest time associated with this process would be at $90^{\circ}(\mathrm{TM})$ due to the greater number of transitions at this angle. From Table 1 no pattern in the results as a function of polarization can be identified. The largest recovery time associated with SHB occurs at an angle of $30^{\circ}$. As already explained the linewidth enhancement factor for SHB is assumed to be zero. ${ }^{12}$ The characteristic power associated with each of the processes did not show a polarization dependence and had magnitudes consistent with other work. ${ }^{17}$ Fig. 8 shows the calculated curves for $\mathrm{CH}$ and $\mathrm{SHB}$, at $60^{\circ}$ and $90^{\circ}$ respectively. A clear variation in the slopes of the $\mathrm{CH}$ curves is visible which is consistent with Table 1 where it can be seen that $\mathrm{CH}$ varies from 819 fs to 668 fs between these two angles. The second couplet of curves in Fig. 8 represent the calculated value for SHB at $60^{\circ}$ and $90^{\circ}$ respectively. There is no visible variation in the slopes of the two curves for these angles, which is also consistent with Table 1, where it can be seen that the variation in SHB is only 7.1 fs between these two angles.

\section{CONCLUSIONS}

A polarization dependent FWM experiment has been carried out to determine if the various gain processes in a bulk SOA are polarization dependent for the tensile strained case. The gain processes considered in this work where CDP, CH and SHB. The faster processes such as TPA were not considered. A polarization dependence of the $\mathrm{CH}$ and SHB was found, leading to the possibility of performing ultra-fast optical switching using the NPR technique. These effects should be much more pronounced in a longer device. Stronger contributions from each effect should also be visible if the FWM was performed closer to the gain peak of the wavelength in the device.

\section{ACKNOWLEDGMENTS}

This work was funded by Enterprise Ireland grant PC/2004/331. 


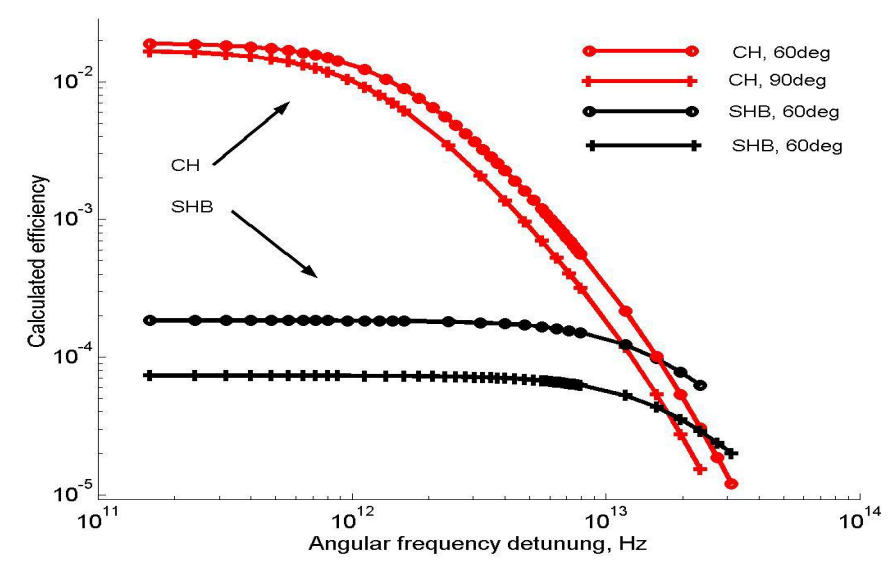

Figure 8. Comparison of calculated curves at $60^{\circ}$ and $90^{\circ}$

\section{REFERENCES}

1. J. Mork, M.L. Nielsen, and T.W. Berg, "The dynamics of semiconductor optical amplifiers," optics and photonic news, pp. 43-48, July 2003.

2. H. Soto, D. Erasme and G. Guekos, "Cross polarization modulation in semiconductor optical amplifiers," IEEE Photonics Tech. Letters Tech. vol.11, pp. pp.970-972, 1999.

3. R.J. Manning, A. Antonopoulos, R. Le Roux, and A.E. Kelly, "Experimental measurement of nonlinear polarisation rotation in semiconductor optical amplifiers," Electronic Letters vol.37(no.4), pp. pp.229-231, 2001.

4. H.J.S. Dorren, D. Lenstra, Y. Liu, M.T. Hill and G. Khoe, "Nonlinear polarization rotation in semiconductor optical amplifiers: Theory and application to all-optical flip-flop memories," IEEE J. Quantum Electronics vol.39(no.1), pp. pp.141-148, 2003.

5. Y. Liu, M.T. Hill, E. Tangdiongga, H. de Waardt, N. Calabretta, G.D. Khoe and H.J.S. Dorren, "Wavelength conversion using nonlinear poilarization rotation in a single semiconductor optical amplifier," IEEE Photonics Tech. Letters Tech. vol.15(no.1), pp. pp.90-92, 2003.

6. D.M. Patrick, A.D. Ellis, D.A.O Davies, M.C. Tatham and G. Sherlock, "Demultiplexing using polarization rotation in a semiconductor laser amplifier," Electronic Letters vol.30(no.4), pp. pp.341-342, 1994.

7. B.F. Kennedy, S. Philippe, P. Landais, A.L.Bradley, and H. Soto, "Experimental investigation of polarization rotation in semiconductor optical amplifiers," IEE Proc. Optoelectronics vol.151(no.2), pp. pp.114-118, 2004.

8. X. Yang, D. Lenstra, G.D. Khoe, and H.J.S. Dorren, "Nonlinear polarization rotation induced by ultrashort optical pulses in a semiconductor optical amplifier," IEEE J. Quantum Electronics vol.223, pp. pp.169-179, 2003.

9. P.Borri, S. Scaffetti, J. Mørk, W. Langbein, J.M. Hvam, A. Mecozzi, and F. Martelli, "Measurement and calculation of the critical pulsewidth for gain saturation in semiconductor optical amplifiers," Optics comm. vol.164, pp. pp.52-55, June 1999.

10. S. Ruiz-Moreno, and J. Guitart, "Practical method for modelling the nonlinear behaviour of a travelling wave semiconductor optical amplifier," IEE proceedings vol.140, pp. pp.39-42, 1993.

11. R.J. Manning, D.A.O. Davies, D. Cotter and J.K. Lucek, "Enhanced recovery rates in semiconductor laser amplifiers using optical pumping," Electronic letters vol.30, pp. pp.787-788, 1994.

12. R.P. Schreieck, M.H. Kwakernaak, H. Jackel, and H. Melchior, "All-optical switching at multi-100-Gb/s data rates with mach-zehnder interferometer switches," IEEE J. Quantum Electronics vol.38(no.8), pp. pp.10531061, 2002.

13. E.P. O'Reilly, A.R. Adams, "Band structure engineering in strained semiconductor lasers," IEEE J. Quantum Electronics vol.30(no.2), pp. pp.366-379, February 1994. 
14. B. Hakki, and T. Paoli, "Gain spectra in GaAs double-heterostructure injection lasers," J. of Applied Physics. vol.46(no.3), pp. pp.1299-1306, 1975.

15. A. Mecozzi, "Four-wave mixing in travelling-wave semiconductor amplifiers," J. Quantum Electronics vol.31, pp. pp.689-699, April 1995.

16. S. Scotti, L. Graziani, A. D’Ottavi, F. Martelli, A. Mecozzi, P. Spano, R. Dall'Ara, F. Girardin and G. Guekos, "Effects of ultrafast processes on frequency converters based on four-wave mixing in semiconductor optical amplifiers," J. Sel. Top. Quantum Electronics vol. 3(no.5), October 1997.

17. F. Girardin, "Contribution a l'etude des phenomenes non-lineaires dans les lasers a semi-conducteurs," $P h D$ Thesis vol.31, pp. pp.93-109, November 1995.

18. J. Buus, "Linewidth broadening in semiconductor lasers - an overview," J. Quantum Electronics vol.23, pp. pp.9-29, 1987.

19. B.F. Kennedy, S. Philippe, M.M. Soto, A.L.Bradley, and P. Landais, "Temporal and spectral dependence on polarization of the input signal in a semiconductor optical amplifier," OSA: Optical amplifiers and their applications, San Francisco, USA, June 2004.

20. S. Murata, A. Tomita, and A. Suzuki, "Influence of free carrier plasma effect on carrier-induced refractive index change for quantum-well lasers," IEEE Photonics Tech. Letters vol.5(no. 1), 1993.

21. L. Schares, C. Schubert, C. Schmidt, H.G. Weber, L. Occhi, and G. Guekos, "Phase dynamics of semiconductor optical amplifiers at 10-40Ghz," IEEE J. Quantum Electronics vol.39, pp. pp.1394-1408, 2003. 